\title{
Microhardness and Indentation Fracture of Potassium Dihydrogen Phosphate (KDP)
}

\section{Introduction}

Potassium dihydrogen phosphate (KDP) is an important electrooptic tetragonal crystal. For example, it is used as a photonic material in the third-harmonic generation (THG) to reduce light wavelength from $1.054 \mu \mathrm{m}$ to $351 \mathrm{~nm}$. Microindentation has been used to measure the Vickers and Knoop hardness of KDP and the resulting cracking on (100) and (001) faces. Hardness anisotropy on the (001) face, or among the (100) and (001) faces, was found to be small (about 20\%). An indentation size effect for both Vickers and Knoop hardness for indenting loads in the range of 25 to $200 \mathrm{~g}$ was observed. The large-load Vickers hardness was estimated as $1.4 \pm 0.1 \mathrm{GPa}$. Anisotropy in the crack sizes on (100) and (001) faces was also observed. Cracks were longer on (100) faces, scaling like $c \sim P^{2 / 3}$; cracks on (001) faces were shorter, scaling like $c \sim P^{1 / 2}$. Assuming elastic and plastic isotropy, crack sizes were analyzed and fracture toughness $K_{c}$ was extracted. An approximate model for analyzing crack-load microindentation data in tetragonal crystals is presented in this article. The model uses the minimum elastic modulus of the material. The effect of the isotropy assumption on the extracted fracture toughness is estimated at about $33 \%$, with a $23 \%$ contribution from elastic anisotropy and $10 \%$ from the slip system plastic anisotropy. Strain-rate effects are identified as important aspects of KDP deformation, especially in laser damage applications.

One of the limiting factors in the use of KDP in THG is its susceptibility to laser damage, a process that couples light absorption with thermal and mechanical effects. (For a summary of the electro-optical properties, see Milek and Neuberger. ${ }^{1}$ )

KDP crystals are water soluble and brittle. Above its ferroelectric Curie temperature ( $123 \mathrm{~K}$ ) the crystal structure of KDP is tetragonal, lacking a center of inversion. KDP is optically uniaxial with the optic axis along the tetragonal $z$ axis or [001] direction. At room temperature the lattice constants are $a=$ $0.7453 \mathrm{~nm}$ and $c=0.6975 \mathrm{~nm}$, as cited in Ref. 1 . The natural habit of crystals grown from solution is a tetragonal prism combined with a tetragonal bipyramid. The prism faces are (100) and (010) planes. The prism axis is [001].
$\mathrm{KDP}$ is relatively soft and brittle as compared to other optical materials, including glasses. In this article microhardness and indentation cracking fracture measurements of KDP indented on crystal planes (100) and (001) are summarized.

Kishan Rao and Sirdeshmukh ${ }^{2}$ measured the Vickers microhardness of KDP at loads of 50 and $100 \mathrm{~g}$, reporting a value of $H_{\mathrm{v}}=1.43 \mathrm{GPa}$ for indentation normal to $\{100\}$ planes (what they called " $a$-direction") and 1.29 GPa for indentation normal to $\{001\}$ planes ("c-direction"). Their error was reported as $\pm 4 \%$. Anbukumar et al..$^{3}$ also measured the Vickers hardness of $\{100\}$ planes of KDP. They reported hardness in the range of 1.77 to $1.57 \mathrm{GPa}$ for loads in the range of 5 to $50 \mathrm{~g}$ and an indentation size effect (ISE) where the hardness decreased with increased load.

Shaskol'skaya et $a l .^{4}$ and Guin et al. ${ }^{5}$ reported measurements of both hardness and cracking in the Vickers measurements of KDP and $\mathrm{KD}_{2 x} \mathrm{H}_{2-2 x} \mathrm{PO}_{4}$ (deuterated KDP, with $x=0$ to 0.95 ). They used loads of 50 to $200 \mathrm{~g}$ and reported a hardness reduction from $1.44 \mathrm{GPa}$ to $1.22 \mathrm{GPa}$ as the extent of deuteration $x$ increased from 0 to 0.95 . Shaskol'skaya et al ${ }^{4}$ also measured the length of cracks (tip-to-tip distance $2 c$ ) due to Vickers indents. They observed that $(2 c) / D$ varied from 3.87 to 3.61 as $x$ increased from 0 to 0.95 . They also reported a value of $51 \mathrm{MPa}$ for the microstrength $P /(2 c)^{2}$ of both KDP and $95 \%$ deuterated KDP.

Guin et al. ${ }^{5}$ reported measurements similar to those of Shaskol'skaya. ${ }^{4}$ They also identified two types of slip systems in KDP: the first system consisted of slip planes (110), (101), (112) and (123) with a common Burgers vector $\langle 111\rangle / 2$; the second slip system was identified as (010)[100].

More recently, Marion ${ }^{6}$ has reported measured values of fracture toughness in KDP crystals. Marion apparently used the direct crack method described by Anstis et al., ${ }^{7}$ although the measured data were not described. Marion ${ }^{6}$ reported fracture toughness $K_{c}$ of $0.2 \mathrm{MPa}^{1 / 2}$, as well as $0.09 \mathrm{MPa} . \mathrm{m}^{1 / 2}$ along the weakest direction (longest crack). No crystallo- 
graphic orientation of the indented faces was reported, however, nor was the applicable elastic constant (modulus) given.

Given the importance of KDP in third-harmonic generation for 351-nm-wavelength laser systems, a systematic study of indentation hardness (Vickers and Knoop) and microindentation cracking in KDP is described below.

\section{Measurements}

Vickers indentation was used to measure the indentation size effect on $H_{\mathrm{v}}$ and also to extract the fracture toughness from the measured dependence of crack size on indenting load. Vickers hardness on (100), (010), and (001) planes of singlecrystal KDP was measured at room temperature with a Tukon Microhardness Tester equipped with a video image-capture camera. Typical descent rate of the indenter is about $1 \mathrm{~mm} / \mathrm{min}$.

The KDP crystal was provided by a commercial vendor and had been grown from the solution. The crystal surfaces were polished by conventional means with nonaqueous slurries to optical standards. Although surface roughness was not directly measured, it was estimated to be approximately 3 to $5 \mathrm{~nm}(\mathrm{rms})$.

The indentation load was in the range of 2 to $200 \mathrm{~g}$, and each load was applied for $15 \mathrm{~s}$. Five indentations were performed at each load. The indentation diagonal $D$ and crack size $2 c$ (tipto-tip distance) were measured with an optical microscope with a 50× objective lens. For the Vickers indentation of (100) and (010) planes, the indenter diagonals were along the principal directions of the type $\langle 100\rangle$. No differences were observed in the indentation diagonal or crack size of (100) and (010) faces.

For the indentation of (001) planes, we selected two indenter orientations: in orientation (1), the indenter diagonals were parallel to [100] and [010]; in orientation (2), the indenter sides were along [100] and [010].

Figure 86.63 shows the measured hardness over the range of indenting loads used. Figure 86.64 shows the measured crack size for Vickers indentation of (100) and (001) faces. The crack-to-indent ratio $c /(D / 2)$ varied from 2 to 4.5 , depending on load and orientation.

Knoop indentation was used to measure the indentation size effect on Knoop hardness and also the hardness anisotropy of the (001) faces. For the indentation size effect (loads of 50 to
$200 \mathrm{~g}$ ), the indenter's long diagonal was along [010]. These results are included in Fig. 86.63.

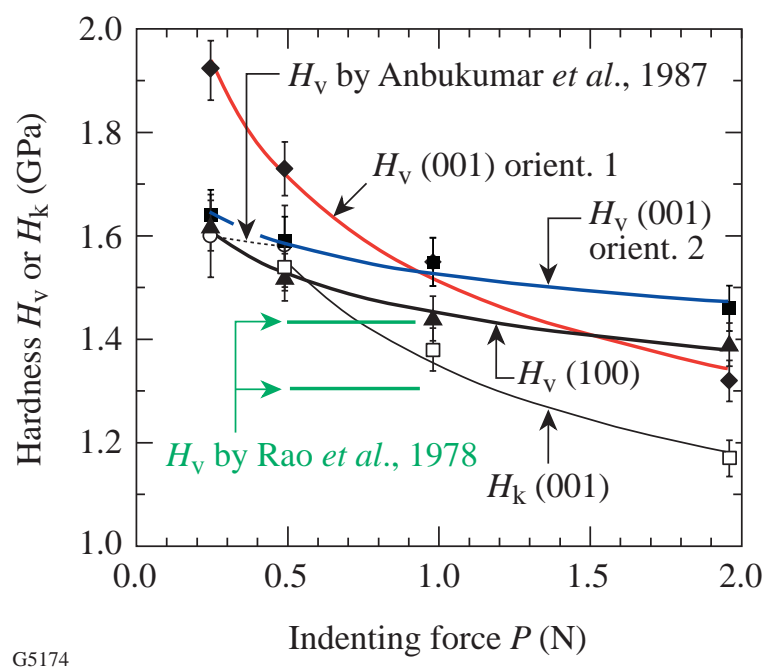

Figure 86.63

Variation of measured hardness (Vickers and Knoop) with load for KDP at room temperature. Vickers indentation was done on faces (100) and on indenter orientations (1) and (2) on the (001) faces. Orientation (1) had the indent diagonals parallel to (100) and (010). Orientation (2) had the indent edges parallel to (100) and (010). Also shown are Vickers measurements by Anbukumar et al. ${ }^{3}$ and Rao et al. ${ }^{2}$ Guin et al. ${ }^{5}$ reported Vickers hardness $1.45 \mathrm{GPa}$ at $200 \mathrm{~g}$.

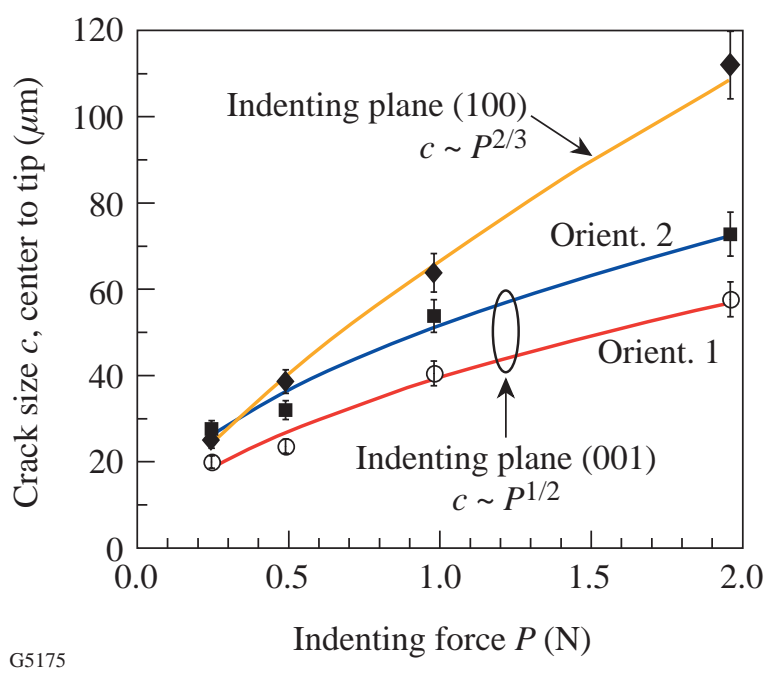

Figure 86.64

Variation of indentation crack size $c$ with indenting load. The tip-to-tip surface crack length is $2 c$. 
For the hardness anisotropy, we used a load of $50 \mathrm{~g}$ and measured the variation of $H_{\mathrm{k}}$ with orientation $\theta$ of the Knoop indenter with respect to the indented surface. Angle $\theta=0^{\circ}$ corresponds to the indenter long diagonal along the [010] direction. The angle $\theta$ was changed in increments of $10^{\circ}$ from $\theta=0^{\circ}$ to $90^{\circ}$. The hardness anisotropy is shown in Fig. 86.65.

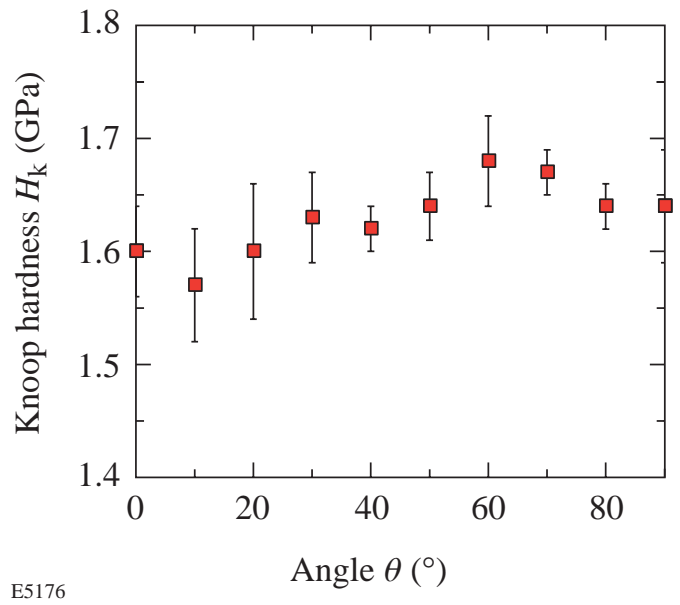

Figure 86.65

Dependence of Knoop hardness on angle $\theta$ of indent on the (001) plane. $\theta=0$ corresponds to the Knoop diagonals being along (100) and (010).

\section{Results}

The measured Vickers hardness is seen to vary between 1.7 and $1.4 \mathrm{GPa}$ over the indenting load range of 50 to $100 \mathrm{~g}$. This hardness range is consistent with the measurements of Rao et al. ${ }^{2}$ over the same load range. Our measurements are also consistent with those of Anbukumar et al..$^{3}$ over the load range of 25 to $50 \mathrm{~g}$, and with Guin et al. ${ }^{5}$ and Shaskol'skaya et al. ${ }^{4}$ who reported a hardness of $1.45 \mathrm{GPa}$ at a load of $200 \mathrm{~g}$.

The measured Vickers hardness brings up two questions: What is the relative hardness of (100) and (001) faces? What is the relative hardness of orientations (1) and (2) of the indenter on face (001)?

Our results show that for loads less than $150 \mathrm{~g}$, (001) faces are harder than (100) faces by as much as $14 \%$ at lower loads. On (001) faces, orientation (1) is harder than orientation (2), by as much as $10 \%$. At loads of about $200 \mathrm{~g}$, however, both faces and both orientations have hardness in the range 1.4 $\pm 0.1 \mathrm{GPa}$; therefore, this value may be used as the load-independent, orientation-insensitive Vickers hardness of KDP.
Our results also show that the Knoop hardness on the (001) face is not strongly anisotropic. The variation of hardness with direction is seen to be less than $10 \%$.

No analysis is available to convert measured microindentation crack sizes to fracture toughness in tetragonal crystals. The only available analysis is for isotropic materials, such as glasses or polycrystalline ceramics (see Ref. 8). Therefore, to convert our direct measurements of indentation crack size to a fracture toughness, we shall assume that KDP can be described by an equivalent isotropic Young's modulus $E$ $=38.7 \mathrm{GPa}$. This value is the mean of the Reuss and Voigt averages for the Young's modulus, with the derivation presented in the Appendix. We have analyzed the microindentation crack measurements (indentation diagonal $D$, tip-to-tip crack size $2 c$ ) using the model of Evans ${ }^{9}$ and Anstis et al. ${ }^{7}$ The comparative merits and applicability of various models to extract the fracture toughness by microindentation cracking in optical glasses and brittle ceramics have been discussed by Ponton and Rawlings ${ }^{10,11}$ and Lambropoulos et al..$^{12}$

Evans ${ }^{9}$ used dimensional analysis and curve fitting over a range of $c /(D / 2)$ from 1.5 to 7 and for many polycrystalline ceramic materials; thus, this model should be applicable to both short and long indentation cracks. According to the Evans model, ${ }^{9}$

$$
\begin{aligned}
K_{c}= & H \sqrt{D / 2}\left(\frac{E}{H}\right)^{0.4} 10^{f(x)}, \quad x=\log _{10}\left(\frac{c}{D / 2}\right), \\
f(x)= & -1.59-0.34-2.02 x^{2} \\
& +11.23 x^{3}-24.97 x^{4}+16.23 x^{5},
\end{aligned}
$$

where $K_{c}$ is the fracture toughness, $H$ is the hardness, $D$ is the indentation diagonal, $E$ is the Young's modulus, and $c$ is the half-crack size. Lankford ${ }^{13}$ included $\mathrm{Al}_{2} \mathrm{O}_{3}$, soda-lime silicate glass, and $\mathrm{NaCl}$ to the materials analyzed by Evans. ${ }^{9}$

Anstis et al. ${ }^{7}$ examined various glasses (glass-ceramic, soda-lime, aluminosilicate, lead alkali), polycrystal $\mathrm{Al}_{2} \mathrm{O}_{3}$ and sapphire, $\mathrm{Si}_{3} \mathrm{~N}_{4}, \mathrm{SiC}, \mathrm{Ca}-\mathrm{PSZ} \mathrm{ZrO}_{2}, \mathrm{Si}$, and $\mathrm{SiC} / \mathrm{Co}$ and concluded that

$$
K_{c}=(0.016 \pm 0.002)\left(\frac{E}{H}\right)^{1 / 2} \frac{P}{c^{3 / 2}}
$$


The Anstis model is based on the assumption that the observed surface cracks are surface traces of sufficiently large radial cracks, so that $c \sim P^{3 / 2}$. On the other hand, the Evans model is applicable for both shorter near-surface cracks, where $c \sim P$, and deeper radial cracks.

As an example of this approach, when the data by Shaskol'skaya et al. ${ }^{4}$ or Guin et al. ${ }^{5}$ are analyzed via the Evans model and with $E=38.7 \mathrm{GPa}$, they yield $K_{c}=0.24 \pm 0.04$ MPa.m ${ }^{1 / 2}$ at the indentation load of $200 \mathrm{~g}$. The Anstis model leads to $K_{c}=0.17 \pm 0.03 \mathrm{MPa} \cdot \mathrm{m}^{1 / 2}$ over the same increase of indentation load. The Anstis model predictions are in agreement with the reported values of 0.09 to $0.20 \mathrm{MPa} . \mathrm{m}^{1 / 2}$ by Marion. ${ }^{6}$ Note, however, that the work of neither Shaskol'skaya et al. ${ }^{4}$ nor Guin et al. ${ }^{5}$ describes the orientation of the indented planes or the orientation of the indenter with respect to the indented plane.

The results of our data analysis using the Evans model are shown in Fig. 86.66, where we have used $E=38.7 \mathrm{GPa}$. We observe that the crack-to-indent aspect ratio $2 c / D$ is in the range of 2 to 4.5 , therefore within the range of applicability of the Evans model. It is seen that the computed fracture toughness $K_{c}$ of indenting the (001) planes is higher than that when indenting the (100) planes. It is also observed that smaller crack sizes apparently produce higher fracture toughness. For $2 c / D$ values of 3 or higher, however, it is seen that the fracture toughness becomes independent of the geometry of the indent producing the cracks. For completeness, Fig. 86.66 shows the (average) \pm (one standard deviation) of the computed fracture toughness for each of the two orientations (1) and (2) on faces (001), as well as that for face (100). The standard deviation was computed from the fracture toughness variation over all the indenting loads used. The results for the two orientations of face (001) overlap, while exceeding that for (100).

The comparisons of the models by Evans ${ }^{9}$ and Anstis et al. ${ }^{7}$ are shown in Fig. 86.67. Both results are based on using Young's modulus $E=38.7 \mathrm{GPa}$. We observe that the Evans model predicts fracture toughness that is a factor of 1.2 to 1.45 higher than the predictions of the Anstis model; however, both models give the same qualitative ranking of the data.

\section{Discussion}

The analysis above rests on two important assumptions. The first assumption is that the anisotropic KDP crystals can be analyzed for fracture toughness using an equivalent isotropic Young's modulus.

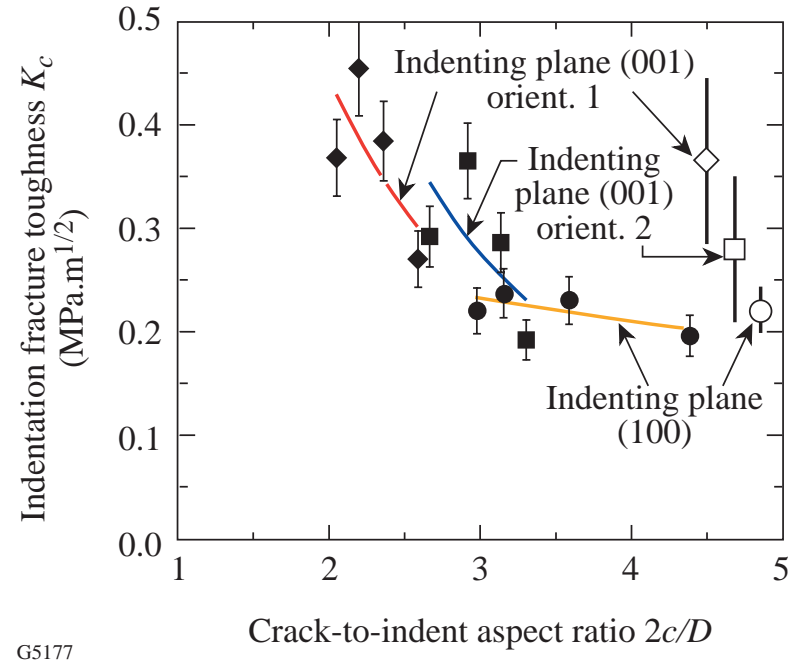

Figure 86.66

Dependence of fracture toughness computed via the Evans $\operatorname{model}^{9}$ on the crack-to-indent aspect ratio $2 c / D$, using the average Young's modulus $E=$ 38.7 GPa. Error bars on the data points reflect measurement uncertainty at each crack size. The thick vertical bars on the right show the (average) \pm (one standard deviation) for each indent orientation. The standard deviations shown on these bars reflect the variation of the fracture toughness over the whole range of indenting loads. To convert these values of fracture toughness to those with the minimum Young's modulus of $E=20.4 \mathrm{GPa}$, multiply these values by 0.774 .

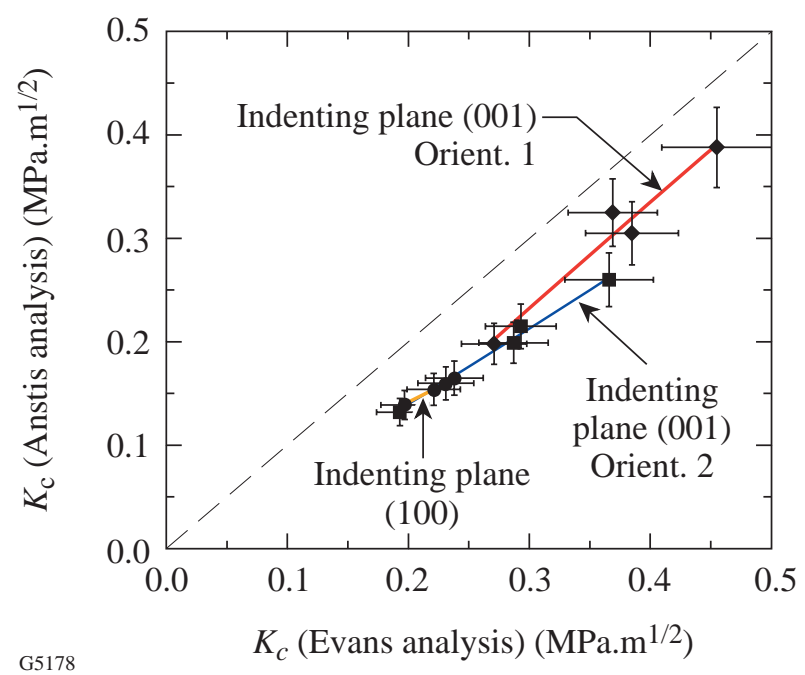

Figure 86.67

Comparison of prediction of fracture toughness by the Evans model ${ }^{9}$ and the Anstis et al. model. ${ }^{7}$ Results are for $E=38.7 \mathrm{GPa}$. The dashed straight line is a line of slope 1 . 
To estimate the effect of such an assumption, for example, on the predictions by the Evans model, we observe that that model uses the term $(E / H)^{0.4}$. As the unconstrained Young's modulus $E$ varies from 20.4 to $65 \mathrm{GPa}$, we conclude that the minimum fracture toughness corresponds to the lowest Young's modulus of $20.4 \mathrm{GPa}$. This, in turn, leads to a change in $K_{c}$ by $(20.4 / 38.7)^{0.4}=0.774$. Therefore, the effect of elastic anisotropy is estimated to be about $23 \%$ on the computed fracture toughness. These results are summarized in Table 86.V. In our data, we give the uncertainty over all the indenting loads used. It is seen that the Anstis et al. model, ${ }^{7}$ when used in conjunction with the minimum Young's modulus of $20.4 \mathrm{GPa}$, yields fracture toughness in the range of $0.09 \pm 0.02$ to $0.22 \pm 0.06 \mathrm{MPa} . \mathrm{m}^{1 / 2}$, in agreement with the values 0.09 to $0.2 \mathrm{MPa} . \mathrm{m}^{1 / 2}$ cited by Marion. ${ }^{6}$

The other important assumption is that the material can be described as an elastic-plastic solid. With a melting point of $T_{m}=525.6 \mathrm{~K}$, the room temperature at which the tests were conducted represents a homologous temperature of 293/525.6 $=0.57$. At such a relatively high temperature, and under the action of the high compressive stresses due to indentation, it is expected that KDP may deform by a variety of mechanisms, including dislocation glide on crystallographic slip systems, or power-law creep by dislocation climb/glide. At temperatures of about $110^{\circ} \mathrm{C}, \mathrm{KDP}$ is known to exhibit macroscopic plasticity in a uniaxial compression. ${ }^{5}$ The room-temperature compressive yield stress does show anisotropy, being $140 \mathrm{MPa}$ for compression along [100], $100 \mathrm{MPa}$ along [110], and $130 \mathrm{MPa}$ for compression along [001]. At $110^{\circ} \mathrm{C}$, these values are reduced by a factor of about $10 ;^{5}$ therefore, anisotropy under uniaxial conditions is about $20 \%$ of the uniaxial compressive yield stress. The anisotropic variation of Knoop hardness that we have measured on the (001) faces was seen to be within $10 \%$ of the average value. Likewise, the largest observed difference in Vickers hardness of (100) and (001) faces was no more than about $10 \%$. Therefore, a total variation of $20 \%$ in hardness due to crystallographic anisotropy is expected, consistent with the anisotropy of the uniaxial compressive yield stress. On the other hand, as Eqs. (3) and (4) show, a $20 \%$ variation in hardness is expected to lead to a variation in the computed fracture toughness of about $10 \%$.

Thus, the estimates of the effects of the Young's modulus anisotropy and hardness anisotropy, when combined, lead to a difference of about $33 \%$ in the fracture toughness as computed by an isotropic elastic-plastic model such as by Evans ${ }^{9}$ or Anstis et al. ${ }^{7}$

On the other hand, at a homologous temperature of 0.57 with respect to the melting point, power-law creep is a timedependent process. Now, the strain rate depends on stress via a power law of exponent in the range of 3 to 8 . In our experiments we have imposed a fixed strain rate, as determined by applying the indentation load for $15 \mathrm{~s}$ on the KDP faces. In typical laser-damage applications, the laser pulse duration over which damage accumulates is of the order of $10 \mathrm{~ns}$, implying, therefore, that the applicable strain rates are much higher than those in indentation.

Table 86.V: Calculated fracture toughness $K_{c}\left(\mathrm{Mpa}^{1 / 2}\right)$ for KDP.

\begin{tabular}{|c|c|c|c|c|}
\hline & \multicolumn{2}{|c|}{ Using average $E=38.7 \mathrm{GPa}$} & \multicolumn{2}{|c|}{ Using minimum $E=20.4 \mathrm{GPa}$} \\
\hline Indents on & Evans model $^{9}$ & Anstis et al. model ${ }^{7}$ & Evans model $^{9}$ & Anstis et al. model ${ }^{7}$ \\
\hline (100) plane & $0.22 \pm 0.02$ & $0.13 \pm 0.03$ & $0.17 \pm 0.02$ & $0.09 \pm 0.02$ \\
\hline $\begin{array}{l}(001) \text { plane, } \\
\text { indent orientation (1) }\end{array}$ & $0.37 \pm 0.08$ & $0.30 \pm 0.08$ & $0.29 \pm 0.06$ & $0.22 \pm 0.06$ \\
\hline $\begin{array}{l}(001) \text { plane, } \\
\text { indent orientation }(2)\end{array}$ & $0.28 \pm 0.07$ & $0.19 \pm 0.06$ & $0.22 \pm 0.05$ & $0.14 \pm 0.04$ \\
\hline Shaskol'skaya et al. ${ }^{4}$ & $0.24 \pm 0.04$ & $0.17 \pm 0.03$ & $0.19 \pm 0.03$ & $0.12 \pm 0.03$ \\
\hline As cited in Marion 6 & & $\begin{array}{l}0.09 \\
\text { ing direct crack meth } \\
\text { t with no information }\end{array}$ & $\begin{array}{l}20 \\
\text { of Anstis et al. } \\
E \text { value used. }\end{array}$ & \\
\hline
\end{tabular}


Given the lack of data describing the dependence on stress and temperature of the deformation mechanisms of KDP, the strain-rate effects are more difficult to estimate. The development of deformation mechanism maps for KDP is thus an area identified for future research.

\section{ACKNOWLEDGMENT}

We wish to thank Prof. Stephen D. Jacobs and Mr. Douglas Smith, both of the Laboratory for Laser Energetics at the University of Rochester, for many discussions and suggestions and sample preparation.

\section{Appendix A: Elastic Anisotropy of KDP}

The elastic behavior of the single-crystal KDP is characterized by six elastic constants, which are shown in Table 86.VI.

Figure 86.68 shows the variation of the Young's modulus of a rod of KDP with orientation of the rod. The figure shows the unconstrained Young's modulus $E_{u}$ (i.e., when the only stress is in the direction of the rod, without any transverse stresses):

$$
\begin{aligned}
\frac{1}{E_{u}}= & \frac{S_{13}}{4}+\frac{S_{44}}{8}+S_{33} \cos ^{4} \theta-\frac{2 S_{13}+S_{44}}{8} \cos 4 \theta \\
& +\left[S_{11}+\frac{\left(-2 S_{11}+2 S_{12}+S_{66}\right)}{4} \sin ^{2} 2 \phi\right] \sin ^{4} \theta,
\end{aligned}
$$

where $\theta$ is the angle between the direction of the rod and the cubic axis [001] and $\phi$ is the angle between the projection of the rod axis on the (001) plane and the [100] direction. The Young's modulus $E_{u}$ varies from about $20 \mathrm{GPa}$ to about $65 \mathrm{GPa}$. When averaged over all rod directions (i.e., integrated over the surface of a unit-radius sphere with differential element of area $d A=\sin \theta d \theta d \phi$ ), we find $\left\langle E_{u}\right\rangle=35.5 \mathrm{GPa}$.

Likewise, the constrained Young's modulus $E_{c}$ (where no strains transverse to the rod are allowed) is

$$
\begin{aligned}
E_{c}= & \frac{C_{13}}{4}+\frac{C_{44}}{2}+C_{33} \cos ^{4} \theta-\frac{C_{13}+2 C_{44}}{4} \cos 4 \theta \\
& +\left[C_{11}+\frac{\left(-C_{11}+C_{12}+2 C_{66}\right)}{2} \sin ^{2} 2 \phi\right] \sin ^{4} \theta ;
\end{aligned}
$$

$E_{c}$ varies from about $40 \mathrm{GPa}$ to $70 \mathrm{GPa}$, as shown in Fig. 86.68. When averaged over all directions, the result is $\left\langle E_{c}\right\rangle$ $=51.4 \mathrm{GPa}$. The results in Fig. 86.68 clearly show that KDP is quite anisotropic.

To get a better idea of the elastic anisotropy, we can also determine the Reuss and Voigt averages as described by Hirth and Lothe, ${ }^{16}$ who summarize the earlier results by Hill. ${ }^{17}$ The Voigt averages for the shear modulus $G_{\mathrm{V}}$ and Lame constant $\lambda_{\mathrm{V}}$ are given by

$$
G_{\mathrm{V}}=\left(3 C_{i j i j}-C_{i i j j}\right) / 30, \quad \lambda_{\mathrm{V}}=\left(-C_{i j i j}+2 C_{i i j j}\right) / 15,
$$

Table 86.VI: Elastic constants of KDP at $20^{\circ} \mathrm{C}$.

\begin{tabular}{|c|c|c|c|c|c|}
\hline \hline$C_{11}$ & $C_{33}$ & $C_{12}$ & $C_{13}$ & $C_{44}$ & $C_{66}$ \\
\hline 71.65 & 56.4 & -6.27 & 14.94 & 12.48 & 6.21 \\
\hline
\end{tabular}

\begin{tabular}{|c|c|c|c|c|c|}
\hline$S_{11}$ & $S_{33}$ & $S_{12}$ & $S_{13}$ & $S_{44}$ & $S_{66}$ \\
\hline 1.51 & 1.95 & 0.18 & -0.40 & 7.81 & 16.2 \\
\hline
\end{tabular}


where repeated indices are summed over the range $i, j=1,2,3$. Here the constants $C_{i j k l}$ relate the stress $\sigma_{i j}$ and strain $\varepsilon_{i j}$ tensors, $\sigma_{i j}=C_{i j k l} \varepsilon_{k l}$. We thus find the average Young's modulus based on the Voigt scheme as $E_{\mathrm{V}}=44.3 \mathrm{GPa}$. The corresponding Poisson ratio is $v_{\mathrm{V}}=0.23$.
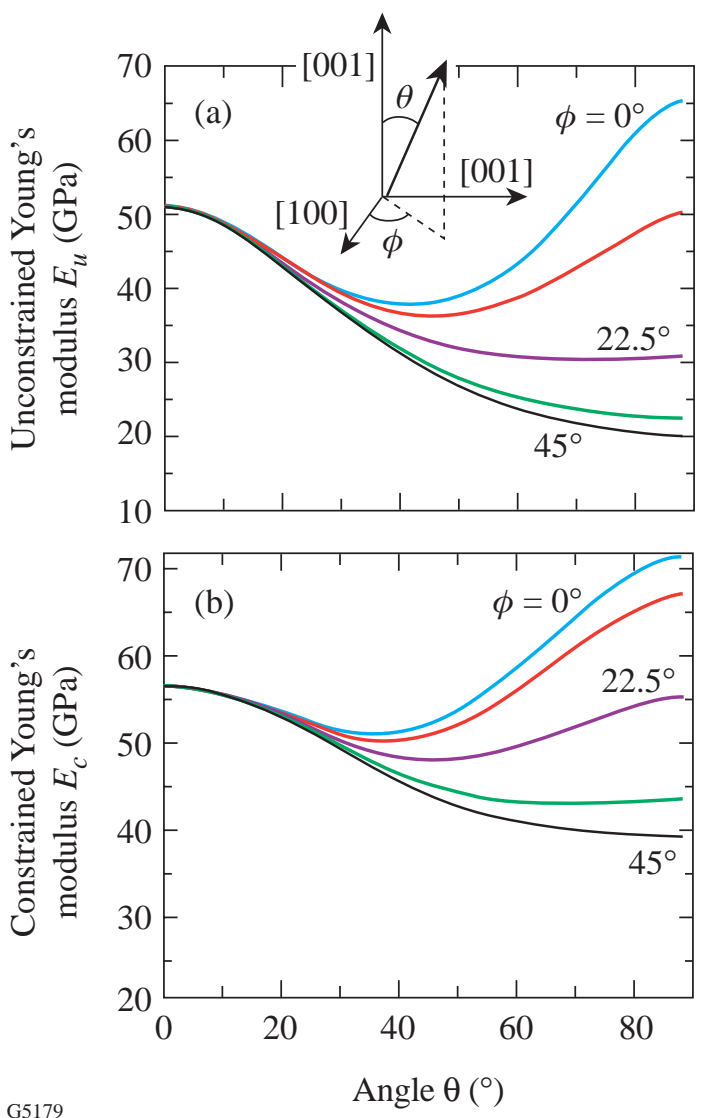

Figure 86.68

Variation of Young's modulus of KDP with crystallographic orientation. The Young's modulus is the ratio of stress to strain for a rod making angles $\theta, \phi$ with the crystallographic axes and stretched in the direction of the rod. Unconstrained $E_{u}$ corresponds to no transverse stresses acting on the rod. Constrained $E_{c}$ is valid when the rod is not allowed to strain in the transverse directions.
The Reuss averages are given by

$$
\frac{1}{E_{\mathrm{R}}}=\left(2 S_{i j i j}+S_{i i j j}\right) / 15, \frac{1}{G_{\mathrm{R}}}=\left(6 S_{i j i j}-2 S_{i i j j}\right) / 15,
$$

where the constants $S_{i j k l}$ relate the strain $\varepsilon_{i j}$ and stress $\sigma_{i j}$ tensors, $\varepsilon_{i j}=S_{i j k l} \sigma_{k l}$. We find the Reuss average of the Young's modulus $E_{\mathrm{R}}=33.0 \mathrm{GPa}$. The corresponding Poisson ratio is $v_{\mathrm{R}}=0.30$.

\section{REFERENCES}

1. S. Anbukumar, S. Vasudevan, and P. Ramasamy, Indian J. Phys. A 61A, 397 (1987)

2. G. R. Anstis et al., J. Am. Ceram. Soc. 64, 533 (1981).

3. R. F. Cook and G. M. Pharr, J. Am. Ceram. Soc. 73, 787 (1990).

4. A. G. Evans, in Fracture Mechanics Applied to Brittle Materials, edited by S. W. Freiman (American Society for Testing and Materials, Philadelphia, 1979), Vol. ASTM STP 678, Part 2, pp. 112-135.

5. C. H. Guin et al., Krist. Tech. 15, 479 (1980).

6. R. F. S. Hearmon, Br. J. Appl. Phys. 3, 120 (1952).

7. S. Haussühl, Z. Kristallogr. 120, 401 (1964).

8. R. Hill, Proc. Phys. Soc. 65A, 349 (1952).

9. J. P. Hirth and J. Lothe, Theory of Dislocations, 2nd ed. (Wiley, New York, 1982), Chap. 13, Sec. 13.2, pp. 424-428.

10. K. K. Rao and D. B. Sirdeshmukh, Indian J. Pure Appl. Phys. 16, 860 (1978)

11. J. C. Lambropoulos, T. Fang, P. D. Funkenbusch, S. D. Jacobs, M. J. Cumbo, and D. Golini, Appl. Opt. 35, 4448 (1996).

12. J. Lankford, J. Mater. Sci. Lett. 1, 493 (1982).

13. J. E. Marion, J. Appl. Phys. 62, 1595 (1987).

14. J. T. Milek and M. Neuberger, Linear Electrooptic Modular Materials, Handbook of Electronic Materials, Vol. 8 (IFI/Plenum, New York, 1972), pp. 177-222.

15. C. B. Ponton and R. D. Rawlings, Mater. Sci. Technol. 5, 865 (1989).

16. ibid., p. 961

17. M. P. Shaskol'skaya, H.-K. Ch'ang, and M. D. Katrich, Izv. Akad. Nauk SSSR Neorg. Mater. 14, 716 (1978). 